\title{
Technical quality and postoperative pain of single visit endodontic treatments of chronic apical periodontitis filled by bioceramic sealer
}

\author{
Seydina O. Niang, ${ }^{1, *}$ Khaly Bane ${ }^{2}$, Mouhamed Sarr ${ }^{3}$, Babacar Touré ${ }^{4}$, Pierre Machtou ${ }^{5}$ \\ ${ }^{1}$ Assistant Professor, ${ }^{2-5}$ Professor, ${ }^{1-4}$ Dept. of Conservative Dentistry \& Endodontics, Cheikh Anta Diop University, Dakar, \\ Senegal, ${ }^{5}$ UFR d'Odontologie, Universitie Denis Diderot, Paris, France
}

\section{*Corresponding Author:}

Email: seydiniang@yahoo.fr

\begin{abstract}
Aims: The aim of this study was to evaluate technical quality and post-operative pain of single visit root canal treatment of chronic apical periodontitis filled by bioceramic sealer.

Material and Methods: Fifty-two non vital teeth with chronic apical periodontitis were treated in single visit. The main inclusion criteria were radiographic evidence of apical periodontitis and a diagnosis of pulpal necrosis. The canals were prepared with ProTaper Universal (Dentsply Maillefer, Ballaigues, Switzerland). The gutta percha master cone was used for final irrigation activation using alternatively $2.5 \% \mathrm{NaOCl}$ and $17 \%$ EDTA. All canals were filled by single cone of gutta-percha (Endosequence BC pointTM, Brasseler, USA) and bioceramic sealer (Endosequence BC sealerTM, Brasseler, USA). The technical quality of root filling was evaluated by control radiographs, the clinical postoperative pain and the need for analgesic consumption were assessed during one week post-treatment. The data were analyzed using the Mann-Whitney $U$ and chi-square test, the significance was set at $\mathrm{P}<.05$

Results: Overall $87 \%$ of all evaluated teeth were found to exhibit a root filling with an acceptable quality level. Assessment of the post-operative sensitivity revealed a rate equal to $6 \%$ (3 cases). The intensity of the pain assessed by a verbal rating scale revealed one case of moderate pain, which required treatment with anti-inflammatories (400 mg ibuprofen) during one week and occlusion reduction.

Conclusion: This study revealed that a single-session endodontic treatment of infected teeth by single-cone root canal obturation associated with bioceramic sealer showed minimal incidence on post-operative pain and acceptable quality level of root filling.
\end{abstract}

Keywords: Apical periodontitis, Bioceramics sealer, Endodontics, Outcomes.

\section{Introduction}

Periapical periodontitis is a chronic inflammatory disease, caused by endodontic infection. Its development is regulated by the host immune/inflammatory response.

The infected root canal is a persistent source of bacterial pathogens, which secondarily stimulate an immune/inflammatory response in the area surrounding the dental root apex, the so-called "periapical" region. ${ }^{1}$

Characteristic features of the periapical lesion are bone resorption and subsequent incomplete wound healing (typically granulomatous tissue formation) in the periapical region. The periapical lesion is an unpleasant outcome of a protective response to the bacteria derived from the infected root canal system, resulting in a chronic inflammation.

Treatment of apical periodontitis consists of performing an etiological endodontic treatment that allows the integrity of the attachment apparatus to be reestablished following reduction of the bacterial load below a sufficient threshold level. ${ }^{2}$

The basic biological rationale aiming ultimate success with root canal treatment consists, primarily on eliminating microorganisms from the entire root canal system and creating a most favorable environment for healing. ${ }^{1}$

Two approaches have been proposed in order to meet this problematic. In one case, residual bacteria are supposed to be eliminated or prevented from repopulating the root canal system by introducing an interappointment dressing during the root canal treatment.

The second approach consists on eliminating the remaining bacteria or rendering them harmless by entombing them in a complete and three-dimensional obturation, finalizing the treatment in one visit, to deprive the microorganisms of nutrition and the space required to survive and multiply.

The introduction of bioceramic root canal sealer is prone to provide an alternative choice and, according to its biologic and adhesive properties, allows to meet the criteria for successful endodontic treatment.

Bioceramics are ceramic materials specifically designed for use in medicine and dentistry. It includes alumina and zirconia, bioactive glass, glass ceramics, coatings and composites, hydroxyapatite and resorbable calcium phosphates, and radiotherapy glasses. ${ }^{3}$

Some calcium silicates (MTA [DENTSPLY Tulsa Dental Specialties] and BioAggregate [DiaDent]) have been used in endodontics as root canal repair materials and for apical retrofills.

Bioceramics are exceedingly biocompatible, nontoxic, do not shrink, and are chemically stable within the biological environment.

It offers clear advantages when used as endodontic sealers: enhanced biocompatibility, possible increased strength of the root following obturation, high $\mathrm{pH}$ (12.9) 
which is strongly antibacterial, during the setting process sealing ability, and ease of use. ${ }^{4}$

In another hand, bioceramics will not result in a significant inflammatory response if an overfill occurs during the obturation process or in case of root repair. A further advantage of the material itself is its ability to form hydroxyapatite and a bond between dentin and the filling material. ${ }^{5,6}$

In addition to their physical and chemical properties, the purpose of bioceramic sealer is to improve the convenience and delivery method, while simultaneously using the water inherent to the dentinal tubules in order to drive the hydration reaction of the material, thereby shortening the setting time.

As materials such as endodontic bioceramic sealer, the single-cone root canal filling technique, although sidelined probably because of a lack of sealing due to the dimensional variations of conventional endodontic cements, is poised to make a comeback.

Furthermore, such a technique, when properly executed, can yield good results due to its ease of use and its capacity to produce high quality obturation results. ${ }^{7}$

However there are very few clinical studies in regard to the benefits of bioceramic sealers use. Most works to date consisted on in vitro studies. ${ }^{8-10}$

The present study was aimed to evaluate the technical quality of root filling and the rate of postoperative pain over a follow-up period of one week after a single visit root canal treatment of teeth with chronic apical periodontitis filled with a bioceramic sealer.

\section{Material and Methods}

This prospective clinical study was carried out at the endodontic clinic of the dental department, located at of medicine pharmacy and dentistry faculty.

The study and informed consent forms were approved by local committee on human research.

\section{Study population}

Study subjects were recruited from the regular pool of patients visiting the endodontic department for root canal treatment.

The patients were recruited by the endodontic investigators following a clinical and radiological diagnosis of chronic apical periodontitis that warranted an endodontic treatment.

Only patients matching the following criteria were included in this study:

1. Patients over 16 years of age;

2. A clinical and radiological diagnosis of chronic apical periodontitis;

3. Informed consent signed by the patient.

An endodontic treatment performed in a single visit was a definitive indication for inclusion of the tooth in the study.

The criteria for non-inclusion were: i) patients exhibiting a periodontal pathology or recent trauma, ii) patients undergoing analgesic or antibiotic medication, iii) patients exhibiting a general pathology with longterm use of medication; iv) endodontic treatment that could not be performed in a single session; v) patients who were not available for follow-ups.

The scoring system proposed by Ørstavik et al. (1986) was used for evaluation of the periapical condition of each tooth. Each of the roots was categorized as: (i) normal periapical structure; (ii) small changes in bone structure; (iii) changes in bone structure with some mineral loss; (iv) Periodontitis with welldefined radiolucent area; and (v) severe periodontitis with exacerbating features.

\section{Intervention}

All of the clinical stages were performed by a single operator, endodontic teacher.

The endodontic treatment was performed in a single visit according to 2006 ESE guidelines ${ }^{11}$ that included pre-endodontic restoration of the missing coronal walls with a glass ionomer cement or a copper band and rubber dam isolation, prior to performing the access cavity with an Endo Access Kit (Dentsply-Maillefer).

The preparation of the root canals system was done using a ProTaper Universal (Dentsply Maillefer, Ballaigues, Switzerland) while using abundant and continuous manual irrigation with $2.5 \%$ sodium hypochlorite. The final irrigation procedure was done with $17 \%$ EDTA activated with a gutta-percha master cone for one minute, followed by abundant rinsing of the canal with $3 \mathrm{ml}$ of $2.5 \%$ sodium hypochlorite in the same manner for 30 seconds. Canals were then dried with sterile paper points.

In regard to the root canal filling, the bioceramic sealer was delivered to the canal using a pre-mixed syringe (Endosequence BC Sealer ${ }^{\mathrm{TM}}$, Brasseler, USA). The gutta-percha master cone (Endosequence BC point $^{\mathrm{TM}}$, Brasseler, USA) was then inserted very slowly into the canal to the working length. The gutta-percha master cone brought sufficient sealing material to the apex. Using a heat source, the gutta-percha cone was thermoplasticized at the orifice canal and then compacted slightly by vertical compaction, and the excess paste removed with a moist cotton pellet.

A control radiograph was done to assess the quality of the root canal filling.

The final step involved restoration of the crown with a composite material, in order to avoid any chance of reinfection of the tooth via the crown, thereby leading to a successful endodontic treatment.

\section{Assessment criteria}

The main assessment criteria were:

1. Radiological ones for the treatment quality (i.e. the density and the limit of the root canal filling),

2. Clinical ones in terms of post-operative sensitivity (flare-ups). 
Radiography assessment of canal obturation quality was performed by two instructors who were not involved in the treatments. Inter examiner agreement, calculated as Cohen's kappa, was 0.80. Any disagreement was resolved through discussion of the issue.

The quality of root fillings was evaluated according to the density of the filling and the distance between the end of the filling and the radiological apex. A filling was considered to be acceptable when no voids or defects along the walls of the canal could be detected and when the filling terminated between 0 and $2 \mathrm{~mm}$ from the radiographic apex.

For teeth with multiple roots, all of the roots were assessed according to the above criteria, and the least satisfactory root determined the quality of the tooth obturation.

The intensity of the pain was assessed using a verbal rating scale (VRS).

0 - No pain

1 - Slight pain/discomfort

2 - Moderate pain relieved by analgesics

3 - Moderate to severe pain not completely relieved by analgesics

4 - Severe pain/swelling not relieved by analgesics and required unscheduled visit

\section{Statistical analyses}

Collection and analysis of the data were carried out with SPSS (version 18.0). The qualitative variables were expressed as percentages, and the quantitative variables as a mean $\pm(\mathrm{SD})$ standard deviation.

Differences between qualitative variable were analyzed with the $\mathrm{Chi}^{2}$ test. The Mann-Whitney $\mathrm{U}$ and chi-square test was used to analyze the association between the variables. The significance level was fixed at $5 \%(\mathrm{p} \leq 0,05)$

\section{Results}

\section{Characteristics of the population}

Forty three consecutive patients (52 cases) were included in this study. The mean age was 31.1 years $+/$ 12.4, with a minimum age of 17 years and a maximum age of 67 . The most commonly encountered age groups were between 17 and 25 years old, followed by 26 to 35 years old.

According to gender this study revealed a predominance of men (69.8\%, i.e 30 patients), compared to women $(30.2 \%$, i.e. 13 patients), i.e a 2.3 sex ratio.

Distribution of the population according to the type of tooth showed that the upper incisors were the most affected 35 teeth (i.e. $67 \%$ of total) followed by molars ( $25 \%$, i.e. 13 teeth), and premolars (8\%, i.e. 4 teeth).

The present study showed that scores of 5 and 4 for the periapical index (PAI) were most encountered, with a percentage of $46.2 \%$ (i.e. 24 cases) and $30.8 \%$ (i.e. 16 cases), respectively. The lower scores of 3 and 2 were less encountered in the present study: $15.4 \%$ (i.e. 8 cases) and $7.7 \%$ (i.e. 4 cases).

\section{Quality of the endodontic treatment}

Assessment of quality of the root canal filling according to the radiological criteria to determine the extent and the density of the root canal filling, showed that $87 \%$ (i.e 45 teeth) of the canal obturations were adequate (Fig. 1).

Assessment of the post-operative sensitivity (Fig. 2) revealed that $94 \%$ of the population (i.e. 49 patients) did not have a complaint, and that only $6 \%$ (i.e. 3 patients) reported some pain lasting from two days to a week. The intensity of pain was assessed using a verbal rating scale that revealed one case of moderate pain that required a one week anti-inflammatory medication (ibuprofen 400) and occlusion reduction.

Two other cases involving low levels of pain did not require medication.

\section{Canal Obturation}

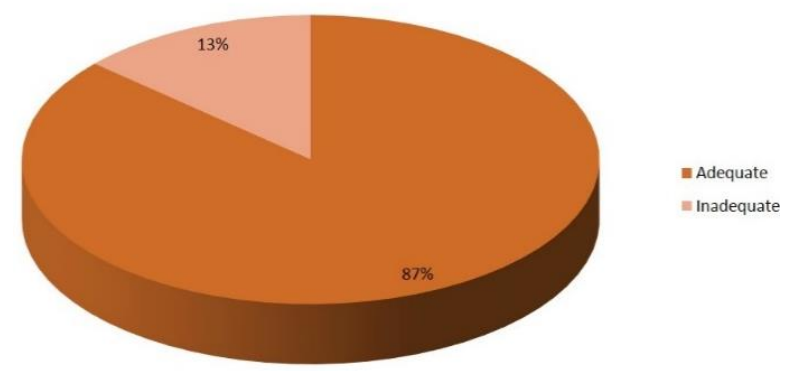

Fig. 1: Quality of root canal filling

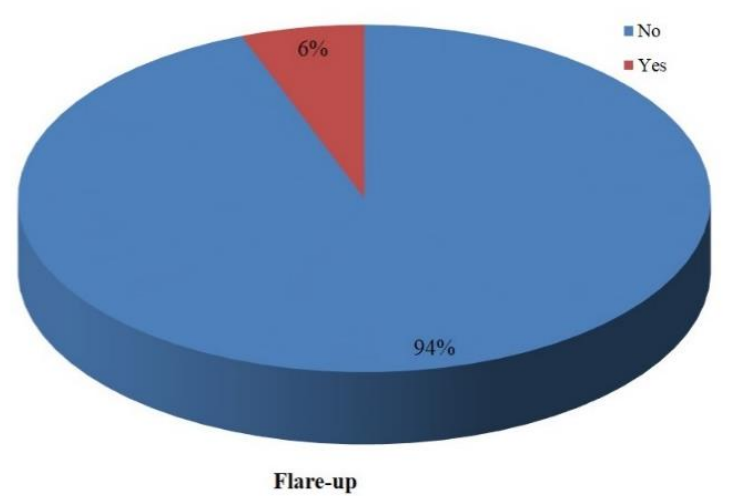

Fig. 2: Post-operative pain

Relationship between the type of tooth and the quality of the endodontic treatment

The distribution of the quality of the root canal filling according the type of tooth showed that $100 \%$ of the obturations of PMs were adequate, while for molars and incisors 84.6 and $85.7 \%$ of the root canal fillings were adequate (Table I). 
Table 1: Association between the type of tooth and the quality of the endodontic treatment

\begin{tabular}{|c|c|c|c|c|c|}
\hline \multirow{2}{*}{\multicolumn{3}{|c|}{ 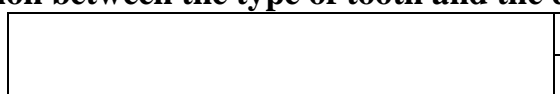 }} & \multicolumn{3}{|c|}{ Canal Obturation } \\
\hline & & & Adequate & Inadequate & Total \\
\hline \multirow{6}{*}{ Type of tooth } & \multirow[t]{2}{*}{ I } & Number & 30 & 5 & 35 \\
\hline & & Percentage & $66.7 \%$ & $71.4 \%$ & $67.3 \%$ \\
\hline & \multirow[t]{2}{*}{ PM } & Number & 4 & 0 & 4 \\
\hline & & Percentage & $8.9 \%$ & $0 \%$ & $7.7 \%$ \\
\hline & \multirow[t]{2}{*}{$\mathrm{M}$} & Number & 11 & 2 & 13 \\
\hline & & Percentage & $24.4 \%$ & $28.6 \%$ & $25.0 \%$ \\
\hline \multicolumn{2}{|l|}{ Total } & Number & 45 & 7 & 52 \\
\hline
\end{tabular}

The distribution of the post-operative sensitivity according to the quality of the obturation did not reveal a significant correlation between the two studied parameters. Two adequate treatments and one inadequate treatment exhibited pain. $\mathrm{p}$-value $=0.299$.

\section{Discussion}

This investigation was a clinical study of a prospective case series aiming assessment of endodontic treatments quality by radiography, and by clinical evaluation of the post-operative sensitivity of teeth with chronic apical periodontitis and treated in a single session. For these teeth, the canal obturation was performed by the single-cone technique in conjunction with bioceramic root canal sealer.

In this study, retroalveolar radiography was used to assess the technical quality of the endodontic treatments, an approach been used by several authors. ${ }^{12,13}$

Based on external the literature and current data, several criteria were adopted to assess quality of the canal obturations. Few authors had only choosen the duration of the canal treatment as criterion. ${ }^{14}$

In the present study, the assessment criteria were the limit and the density of the canal filling, which are in line with most authors' works. ${ }^{13,15}$

The studied population was comprised of 52 patients.

On average, they were 31.1 years of age $+/-12.4$, with a minimum of age of 17 years and a maximum of 67 years. The most commonly encountered age group was those between 17 and 25 years followed by 26 and 35 year.

The distribution of the population according to gender revealed a predominance of men $(69 \%)$ compared to women (31\%) with a sex-ratio (men/women) of 2.19.

The predominance of men could be explained by the fact that the majority of teeth showing chronic apical periodontitis treated in the present study were consecutive to an old tooth injury, that males are more exposed. Indeed, gender is a risk factor for extrinsic alveolar dental injuries.

Assessment of the quality of root canal fillings in the present study, according to the radiological criteria to determine the extent and the density of canal obturations, showed that $87 \%$ of the root canal fillings were adequate.

Studies in regard to the quality of endodontic treatments performed by specialists are nearly entirely lacking from the literature. The majority of endodontists mainly report the success rate or survival of their treatments, which are generally higher than the level of adequate treatments.

One of the rare published studies referring specifically to quality in regard to endodontic treatments was performed by six endodontists in Melbourne (Australia), ${ }^{16}$ and showed that on a sample of 1,351 root canals, homogeneity and adequate density were found along the entire length of the canal in $88.6 \%$ of cases.This is very close to the value found in the present study.

These values, however, are well above the trends seen in the endodontic literature when the treatments are often performed by general dental practitioners. Thus, Toure et al. ${ }^{17}$ have shown that only $17.7 \%$ of the endodontic obturations in a Senegalese sub-population were technically acceptable.

The study by Boucher et al. ${ }^{18}$ revealed a low level of quality in France, with only $21 \%$ of the obturations being deemed to be technically acceptable.

This pronounced difference could be explained by the demanding nature of the surgical procedure requiring skill and time. Moreover, the material used in this study i:e bioceramic sealer (calcium silicate) exhibits numerous physical, chemical, and biological properties that allow for a good match with the aims of the endodontic treatment. Indeed, its high $\mathrm{pH}$ during setting, biocompatibility, and dimensional stability provides key advantages over conventional obturation sealers.

According to Zhang et al., ${ }^{9}$ bioceramic sealer is alkaline (pH above 12), providing a strong anti-bacterial activity that can eliminate Enterococcus faecalis in a two minute exposure, thus underscoring it usefulness in single-session endodontic treatments.

The quality of the sealer was also investigated by Nagas et al. ${ }^{10}$ The hydrophilic sealer has an excellent flow and forms hydroxyapatite which allows it to bond instantly to both the dentin and to the bioceramic nano particles-coated cones (e.g. BC Points ${ }^{\mathrm{TM}}$ ) creating thus a full seal with no voids. 
A recent study has shown that bioceramics, when used with impregnated and coated cones, increase the resistance to root fractures and lead to a quality level comparable to that of a live tooth. ${ }^{19}$

The excellent flow of the sealer was also studied by Zhang et al, ${ }^{9}$ who found that due to its extremely small particles and its hydrophilic nature, BC Sealer readily fills all of the canal's anatomical space.

The single-cone obturation technique offers advantages since, when used with an appropriate material such as bioceramics, is consistent with most common obturation techniques such as warm vertical and cold lateral compactions. In addition to its simplicity, and significant time saving, its low cost avoids the need for specific expenditures.

Assessment of the post-operative sensitivity has showed that $94 \%$ of the population had no complaints, and that only $6 \%$ reported sensitivity that ranged from two days to a week.

This post operative pain rate was independent of the quality of the treatment and was associated neither with the type of tooth nor with the initial peri-apical status. This rate is very low compared to the data found in the literature. Indeed, in a recent study, ${ }^{20} 32 \%$ of the singlesession endodontic treatments of non-vital teeth with bone rarefaction were associated with painful symptoms, among which $24 \%$ were minor (insignificant) and $8 \%$ were moderate (significant). No case of severe pain was reported.

Back to the 80 's, Roane et al. ${ }^{21}$ have shown a twofold higher rate of reported pain following endodontic treatments performed in multiple sessions compared to those done in a single session.

In 2011, Eleazar et al. compared the rate of flare-ups following endodontic treatments of molars with necrotic pulp, and concluded that single-visit endodontic treatments had an advantage over treatments involving multiple sessions. ${ }^{22}$ The low rate of post-operative pain in the present study may hence be explained in one hand, by the single-session endodontic therapeutic approach. Furthermore, the biological properties of the bioceramic canal obturation materials could also have played a role.

Although Bc sealer has been shown to exhibit minimal toxicity, some precautions need to be taken however to avoid over extension of the material. ${ }^{23-24}$

Nowadays, bioceramic materials have become part of dentistry armamentarium, mainly due to their high level of biocompatibility and their potent anti-bacterial activity. ${ }^{23-25}$

\section{Conclusion}

This study revealed that single-session endodontic treatment of infected teeth is readily achievable when carried out with a rigorous surgical protocol respecting the scientifically derived standards. Furthermore, when carried out with appropriate materials such as bioceramics (e.g. Bc sealer, Bc point), the single-cone canal obturation technique has the potential to become a widely used procedure in light of its ease-of-use and low cost.

Yet further studies with a higher level of evidence, such as randomized clinical trials, are required to better appreciate the clinical benefits of bioceramic obturation materials in endodontics.

Funding: No funding sources.

Conflict of interest: None declared.

\section{References}

1. Alley BS, Kitchens GG, Alley LW, Eleazer PD. A comparison of survival of teeth following endodontic treatment performed by general dentists or by specialists. Oral Surg Oral Med Oral Pathol Oral Radiol Endod 2004;98(1):115-8.

2. Siqueira JF Jr, Rôças IN. Clinical implications and microbiology of bacterial persistence after treatment procedures. J Endod 2008;34(11):1291-301.

3. Best S.M, Porter A.E, Thian E.S, Huang J. Bioceramics: past, present and for the future. J Eur Ceramic Society 2008;28:1319-27.

4. Silva Almeida LH, Moraes RR, Morgental RD, Pappen FG. Are Premixed Calcium Silicate-based Endodontic Sealers Comparable to Conventional Materials? A Systematic Review of In Vitro Studies. J Endod 2017;43(4):527-35.

5. Candeiro GT, Moura-Netto C, D'Almeida-Couto RS, Azambuja-Júnior N, Marques MM, Cai S, Gavini G. Cytotoxicity, genotoxicity and antibacterial effectiveness of a bioceramic endodontic sealer. Int Endod J 2015.

6. Ruparel NB, Ruparel SB, Chen PB, Ishikawa B, Diogenes A. Direct effect of endodontic sealers on trigeminal neuronal activity. J Endod 2014;40(5):683-7.

7. Koch K, Brave D. A New Endodontic Obturation Technique. Dent Today 2006;25(5):102-7.

8. Zhou HM, Du TF, Shen Y, Wang ZJ, Zheng YF, Haapasalo M. In vitro cytotoxicity of calcium silicatecontaining endodontic sealers. J Endod 2015;41(1):56-61.

9. Zhang H, Shen Y, Ruse ND, Haapasalo M. Antibacterial activity of endodontic sealers by modified direct contact test against enterooccus faecalis. $J$ Endod 2009;35(7):1051-5.

10. Nagas E, Uyanik MO, Eymirli A, et al. Dentin moisture conditions affect the adhesion of root canal sealers. $J$ Endod 2012;38(2):240-4.

11. European Society of Endodontology. Quality guidelines for endodontic treatment: consensus report of the European Society of Endodontology. Int Endod J 2006;39(12):921-30.

12. Tordai B, Schreindorfer K, Lempel E, Krajczár K. Factors affecting ultrasonic removal of separated endodontic instruments: A retrospective clinical study. Quint Int 2018;49(4):257-66.

13. Colombo M, Bassi C, Beltrami R et al. Radiographic technical quality of root canal treatment performed by a new rotary single-file system. Ann Stomatol (Roma) 2017;8(1):18-22.

14. Burke FM, Lynch CD, Ní Ríordáin R, HANNIGAN A. Technical quality of root canal fillings performed in a dental school and the associated retention of root-filled teeth: a clinical follow-up study over a 5 -year period. $J$ Oral Rehabil 2009;36(7):508-15.

15. Segura-Egea JJ, Jiménez-Pinzón A, Poyato-Ferrera M, Velasco-Ortega E, Ríos-Santos JV. Periapical status and 
quality of root fillings and coronal restorations in an adult Spanish population. Int Endod $J$ 2004;37(8):525-30.

16. Bierenkrant DE, Parashos P, Messer HH. The technical quality of nonsurgical root canal treatment performed by a selected cohort of Australian endodontists. Int Endod J 2008;41(7):561-70.

17. Touré B, Kane AW, Sarr M, Ngom CT, Boucher Y. Prevalence and technical quality of root fillings in Dakar, Senegal. Int Endod J 2008;41(1):41-9.

18. Boucher Y, Matossian L, Rilliard F, Machtou P. Radiographic evaluation of the prevalence and technical quality of root canal treatment in a French Subpopulation. Int Endod J 2002;35(3):229-38.

19. Ghoneim AG, Lutfy RA, Sabet NE, Fayyad DM.

Resistance to fracture of roots obturated with novel canalfilling systems. J Endod 2011;37(11):1590-2.

20. Sumita Bhagwat, Deepil Mehta. Incidence of postoperative pain following single visit endodontics in vital and non-vital teeth: An in vivo study. Contemp Clin Dent 2013;4(3):295-302.

21. Roane JB, Dryden JA, Grimes EW. Incidence of postoperative pain after single- and multiple-visit endodontic procedures. Oral Surg Oral Med Oral Pathol 1983;55(1):68-72.
22. Elsayed RO, Abu-Bakr NH, Ibrahim YE. Quality of root canal treatment performed by undergraduate dental students at the University of Khartoum, Sudan. Aust Endod J 2011;37(2):56-60.

23. Loushine BA, Bryan TE, Looney SW, et al. Setting properties and cytotoxicity evaluation of a premixed bioceramic root canal sealer. J Endod 2011;37(5):673-7.

24. Zoufan K, Jiang J, Komabayashi T, Wang Y, Safavi KE, Zhu Q. Cytotoxicity évaluation of Gutta Flow and Endo Sequence BC sealers. Oral Surg Oral Med Oral Pathol Oral Radiol Endod 2011;112(5):657-61.

25. Damas BA, Wheater MA, Bringas JS, Hoen MM. Cytotoxicity comparison of minéral trioxide aggregates and EndoSequence bioceramic root repair materials. $J$ Endod 2011;37(3):372-5.

How to cite the article: Niang S., Bane K., Sarr M., Touré B., Pierre Machtou P. Technical quality and postoperative pain of single visit endodontic treatments of chronic apical periodontitis filled by bioceramic sealer. IP Indian J Conserv Endod 2018;3(3):92-97. 